\title{
Anatomic guided ablation of the atrial right ganglionated plexi is enough for cardiac autonomic modulation in patients with significant bradyarrhythmias
}

\author{
Dinis Mesquita ${ }^{1}$, Leonor Parreira ${ }^{1}$, Pedro Carmo ${ }^{2}$, Rita Marinheiro ${ }^{1}$, Diogo Cavaco ${ }^{2}$, Pedro \\ Amador $^{1}$, Elisabete Vaz $^{2}$, Francisco Costa ${ }^{2}$, José Farinha ${ }^{1}$, Mauricio Ibrahim Scanavacca ${ }^{3}$, \\ Rui Caria ${ }^{1}$, and Pedro Adragão ${ }^{2,4}$ \\ ${ }^{1}$ Hospital de S. Bernardo, Centro Hospitalar de Setúbal E.P.E. \\ ${ }^{2}$ Hospital de St. ${ }^{a}$ Cruz, Centro Hospitalar de Lisboa Ocidental E.P.E. \\ ${ }^{3}$ Hospital de St. ${ }^{\text {a }}$ Cruz, Centro Hospitalar de Lisboa Ocidental, E.P.E. \\ ${ }^{4}$ EP Centre Cardiology, Hospital de Santa Cruz
}

July 1, 2020

\begin{abstract}
Introduction: Cardiac autonomic system modulation by endocardial ablation targeting atrial ganglionated plexi (GP) is an alternative strategy in selected patients with severe functional bradyarrhythmias, although no consensus exists on the best ablation strategy. The aim of this study was to evaluate if a simplified approach by a purely anatomical guided ablation of just the atrial right GP is enough for the treatment of these patients. Methods: We prospectively enrolled patients with significant functional bradyarrhythmias and performed endocardial ablation purely guided by 3D electroanatomic mapping directed at the atrial right GP and accessed parameters of parasympathetic modulation and recurrence of bradyarrhythmias. Results: Thirteen patients enrolled (76.9\% male, median age 51, 42-63 years). After ablation, a median RR interval shortening of 28.3 (25.6$40.3) \%$ occurred $(111,937.5-1395.4 \mathrm{~ms}$ to $722.9,652.2-882.4 \mathrm{~ms}, \mathrm{p}=0.0015)$. The AH interval also shortened $(19,10.5-35.7 \%)$ significantly after the procedure $(115,105-122 \mathrm{~ms}$ to $85,71-105 \mathrm{~ms}, \mathrm{p}=0.002)$ as well as Wenckebach cycle length $(11.1,5.9-17.8 \%$ shortening) from 450,440-510ms to 430,400-460ms, $\mathrm{p}=0.0014$. On 24-hour Holter monitoring there was significant increase in heart rates (HR) of patients after ablation (minimal HR increased from 34 (26-43)bpm to 49 (43-56)bpm, p=0,0063 and mean HR from 65 (47-72) bpm to 78 (67-87) bpm, $\mathrm{p}=0.0015)$. No patients had recurrence of symptoms or significant bradyarrhythmias during a median follow-up of 8.4 months. Conclusions: A purely anatomic guided procedure directed only at the atrial right ganglionated plexi seems to be enough as a therapeutic approach for cardiac parasympathetic modulation in selected patients with significant functional bradyarrhythmias.
\end{abstract}

\section{Introduction:}

Bradyarrhythmias manifested as sinus arrest, sinus bradycardia, brady-tachy syndrome, transient atrioventricular (AV) block or cardioinhibitory syncope can be associated with autonomic imbalance, for which parasympathetic drive predominates. In these cases, specially in younger patients, this poses a conundrum when it comes to decisions whether and how to treat. Increasing evidence, also states a role of vagal tone in some patients with atrial fibrillation (AF), as parasympathetic stimulation is known to shorten atrial effective refractory period and increases the probability of multiple reentrant circuits in the atrial myocardium, therefore increasing stability of atrial fibrillation ${ }^{(1,2,3)}$. An increasing number of studies ${ }^{(3,4,5,6,7,8,9)}$ have convincingly shown that parasympathetic cardiac modulation is an alternative and effective therapeutic method for the treatment of these patients, although there is no standardized technique or endpoint criteria to assess therapeutic success. 
The objective of this study was to understand if performing a simplified, purely anatomic guided ablation aiming only at the atrial right GP proves to be a valid and successful strategy to perform cardiac modulation in patients with significant functional bradyarrhythmias.

\section{Methods:}

\section{Study population}

Between February 2017 and October 2019, we prospectively enrolled 13 patients in three hospital centers, with documented episodes of severe functional bradyarrhythmias suggestive of vagal etiology (sinus bradycardia and / or arrest, brady-tachy syndrome, transient AV block and cardioinhibitory syncope). Functional bradyarrhythmias were acknowledged after exclusion of reversible causes, such as negative chronotropic drugs, ionic disorders, thyroid dysfunction, cardiac ischemia, obstructive sleep apnea, intrinsic sinus or AV node disease and in patients engaged in competitive sports, after deconditioning. Intrinsic sinus and AV node disease were excluded after assessment of a positive chronotropic response on 24-hour Holter monitoring and on exercise treadmill test, with absence of exercise induced AV block and when bradyarrhythmias were suggestive to occur in a vagal setting as during sleep, post meals or prolonged standing. Patients with suspected vagal syncope underwent tilt testing that exhibited syncope with predominant cardioinhibitory response. An ECG was performed in all patients and the HR and PQ interval was measured. Patients with abnormal ECG apart from sinus bradycardia, first-degree or second-degree Mobitz type I AV block or with abnormal transthoracic echocardiogram were excluded.

Included patients were either symptomatic (syncope / pre-syncope related to bradycardia) or had severe bradycardia with an indication for pacing regardless of symptoms as outlined in the current European Society of Cardiology Guidelines for the management of bradyarrhythmias ${ }^{(10)}$.

\section{Baseline electrophysiological study}

The procedure was performed in fasting state, under general anesthesia in 5 patients (standard procedure in one center) for which propofol and atracurium were used during the study. In the remaining procedures, patients were under conscious sedation with propofol and remifentanil and monitored with bispectral index (BIS@). Three right femoral vein punctures allowed introduction of a $7 \mathrm{~F}$ diagnostic decapolar catheter in the coronary sinus, a $6 \mathrm{~F}$ diagnostic quadripolar catheter at His location and one 8.5F SL1@ sheath (Abbot Inc.) Baseline electrophysiological study was performed using a multichannel cardiac recorder for bipolar intracardiac electrograms with filtered signals at 30-300 Hz (EP WorkMate Claris System@, Abbott Medical and LabSystem Proß, Boston Scientific), displaying 3 surface ECG leads (DII, aVF and V1) and with programmed digital stimulation (EP-4@ Cardiac Stimulator, Abbott Medical and Qubic Stim, Biotronik). Baseline intervals were measured, and sinoatrial node function was evaluated by measuring RR intervals and AV node function was assessed by measuring Wenckebach cycle length (WBCL) (atrial decremental stimulation at which Wenckebach AV block occurs).

\section{GP mapping and ablation in the left atrium and AF ablation}

We performed endocardial electroanatomic mapping of the left atria (LA) for anatomic geometry recon-

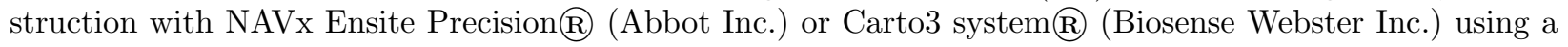

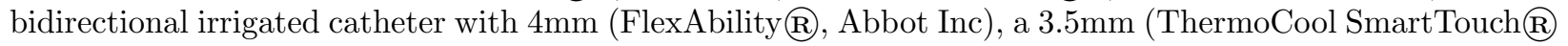
or Navistar RMT ThermoCool@, Biosense Webster Inc.) or a circular mapping catheter in patients who

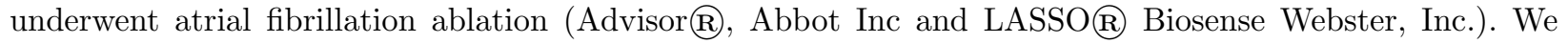

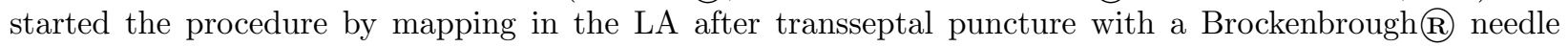
(Medtronic Inc.) under fluoroscopic guidance. After the puncture a bolus o $10000 \mathrm{IU}$ of heparin was administrated and targeted activated clot time (ACT) was maintained between 300-400 seconds and monitored every 30 minutes after achieving a value of 300 seconds. We identified the pulmonary vein (PV) ostia, left atrial appendage and mitral valve annulus. In the reconstructed anatomy, we marked the empiric anatomic location of the atrial right GP site as previously described in the literature ${ }^{(1,4,5,9,11)}$ (Figure 1$)$. The anterior right GP in the common vestibulum of the anterior aspect of the right pulmonary veins and the inferior right 
GP in the inferior aspect of the inferior pulmonary vein).

For ablation of the GP, we used only an anatomical approach aiming at previously described empiric GP sites. Radiofrequency (RF) lesions were performed for 30 seconds with $25 \mathrm{~W}$ (20W in the inferior aspect of the right inferior pulmonary vein) with a set temperature of $43^{\circ} \mathrm{C}$, approximately, $10 \mathrm{~mm}$ from the respective PV in a cloud-like shape. The endpoint of the ablation was the deployment of lesions in all pre-specified ablation targets, irrespective of any change in RR interval or Wenckebach Cycle Length (WBCL).

For patients with an indication for AF ablation, the used technique was $\mathrm{PV}$ isolation (PVI), with RF circumferential lesions at PV antrum (around 10-15mm from PV ostia) with $20 \mathrm{~W}$ for 20 seconds in the posterior wall and $25 \mathrm{~W}$ for 30 seconds in the remaining walls, with a limit temperature of $43^{\circ} \mathrm{C}$ and aiming at abolishment of local electrograms to $<0,1 \mathrm{mV}$. The endpoint for $\mathrm{PV}$ isolation success was the disappearance or dissociation of PV potentials and the achievement of bidirectional block between PV and LA. If necessary, additional RF lesions were made at conduction gap sites.

\section{GP mapping and ablation in the right atrium}

After LA ablation, the catheter was withdrawn to the right atrium (RA) and the anatomy of the RA was obtained. The superior and inferior vena cava, the interatrial septum and coronary sinus ostium were identified. In the RA (Figure 2) we marked the empiric location of the right aspect of GP, opposite to the area of the LA GP ablation (Figure 3), at the septal aspect of the superior vena cava junction and the posterior aspect of the interatrial septum (between the posterior wall and the coronary sinus ostia) ${ }^{(1)}$. In the RA we made RF lesions for 30 seconds at $35 \mathrm{~W}$ and a temperature of $43^{\circ} \mathrm{C}$. The endpoints were as on the LA, the deployment of lesions in all pre-specified ablation sites at the specified described parameters.

\section{Post Ablation Electrophysiological study}

The basic intervals, RR intervals and WBCL were measured using the same approach as in the baseline electrophysiologic study.

\section{Follow up}

Immediate complications of the procedure were assessed, namely vascular access complication, pericardial effusion, stroke or arrhythmias. All patients were scheduled for presential follow-up at 30 days with ECG with measurement of HR and PQ interval. At the six-month visit and every six months afterwards a 24hour Holter monitoring was performed. Three patients had an implantable cardiac monitor (ICM) implanted (for which two were implanted at the same admission of the ablation procedure, at physician discretion). Antiarrhythmic drugs were maintained for at least 3 months in patients with AF and ceased afterwards if no recurrences were detected.

\section{Statistical Analysis}

All analysis was performed using version 12 of STATA $\cap$ (Statistics Data Analysis). Data is presented as median and lower and upper quartiles (Q1-Q3) for continuous variables and as absolute number and percentage for categorical variables.

Continuous variables were compared with the use of the Wilcoxon rank test for repeated measurements. A value of $\mathrm{p}<0.05$ was considered statistically significant.

\section{Ethics Disclosure}

This study complies within declaration of Helsinki rules. All patients signed the informed consent and the study was approved by the ethical committee of the three hospitals.

\section{Results:}

\section{Study population}


We enrolled 13 patients, 10 males (76.9\%), with a median age of 51 (42 - 63) years. Nine patients $(69.2 \%)$ were symptomatic (syncope / pre-syncope related to bradyarrhythmia) and the remaining four patients were asymptomatic (\#2, \#6, \#10 and \#11) (table 1). The diagnosis was transient high-grade AV block in three patients (one had also sinus arrest), cardioinhibitory syncope in two patients and sinus arrest in eight patients. The mean ECG heart rate (HR) was 55 (45 - 61), beats per/minute (bpm) (table 2) and the PQ interval on 12 lead ECG among patients was 180 (170 - 220) ms. Four out of eight patients with sinus arrest had also sinus bradycardia and four had brady-tachy syndrome with paroxysmal atrial fibrillation (table 1).

The longest pause (either for sinus arrest or AV block) was 7000 (4000 - 10000) ms. Regarding assessment on 24-hour Holter monitoring, minimal HR was 34 (26 - 43) bpm and mean HR 65 (47 - 72) bpm.

\section{Baseline electrophysiological study}

All patients were in sinus rhythm at the day of the procedure. The measured AH interval was 115 (105 122) $\mathrm{ms}$ and HV interval were 45 (45-52) ms. The measured RR intervals were 1111 (937.5 - 1395.4) ms and the WBCL 450 (440 - 510) ms.

\section{GP mapping and ablation and AF ablation}

In all patients, successful access to LA and performance of 3D electroanatomic mapping was possible, with identification of PV ostia and GP specified anatomic location sites. Completion of the lesion subset prespecified to ablate right GP was undertook in all patients, with approaches from the left and right atria. In the four patients with documented paroxysmal atrial fibrillation, PV isolation was done and with achievement of bidirectional block in all patients.

\section{Post Ablation Electrophysiological study}

Measured AH interval (85, 71 - $105 \mathrm{~ms})$ significantly decreased after GP ablation ( $\mathrm{p}=0.002)$, with a shortening of $19(10,5-35,7) \%$. No differences were observed in $\mathrm{HV}$ interval $(45,45-50 \mathrm{~ms}, \mathrm{p}=0.08)$. After ablation, RR intervals were $722.9(652.2-882.4) \mathrm{ms}$, significantly reduced $(\mathrm{p}=0.0015)$, and with a shortening of 28.3 $(25.6-40.3) \%$ (Figure 4). We also encountered a significant difference between pre and post ablation WBCL $(430,400-460 \mathrm{~ms}, \mathrm{p}=0.0014)$ with a correspondent shortening of $11.1(5-9-17.8) \%$ (table 3$)$.

\section{Follow up}

No immediate or delayed complications of the procedure were observed.

The effect of parasympathetic modulation led to a significant increase of HR to $80(65-85)$ bpm, $\mathrm{p}=0.007$ and a reduction of PQ intervals in 12 lead ECG at 30 days to $160(150-180) \mathrm{ms}, \mathrm{p}=0.002$. On 24-hour Holter monitoring there was a significant increase in minimal HR to $49(43-56) \mathrm{bpm}, \mathrm{p}=0,0063$ and in mean to HR $78(67-87) \mathrm{bpm}, \mathrm{p}=0,0015$ (table 2).

After a median follow-up of $8.4(6.5-23.4)$ months, no patient had recurrence of symptoms and there was no recurrence of sinus arrest among the cohort. One patient referred for ablation for transient high-grade AV block showed paroxysmal $2^{\text {nd }}$ degree Mobitz type I AV block (patient \#10) and the remaining patients had no evidence of recurring AV block. None of the patients needed pacemaker implant. No significant atrial arrhythmias or AF recurrence were detected during follow-up apart for one patient without previously documented AF with ICM that had one-hour episode of AF at two months post ablation (patient \#6).

\section{Discussion:}

Although current guidelines recommend pacemaker implant in patients with severe symptomatic bradyarrhythmias, there is evidence that some of these disorders are related to functional imbalance in cardiac autonomic nervous system with a predominant excess of vagal tone ${ }^{(10)}$. Cardiac parasympathetic drive occurs through efferent signals from vagal fibers, connected to postganglionic cells within atrial walls or in para-cardiac ganglia, mainly by decreasing automatism, excitability and conductivity. Cardiac modulation of parasympathetic GP abolishes the influence of excessive parasympathetic autonomic influence ${ }^{(4)}$. Even though GP are located in epicardial fat pads, the extensive network of intramural atrial micro ganglia ${ }^{(2,7,12)}$ 
renders endocardial ablation effective. Various reports ${ }^{(4,5,7,12)}$ have shown that modulation of cardiac parasympathetic system supports this approach as an alternative strategy for these patients, particularly at younger ages for whom pacemaker implant is unwanted due to its potential lifelong complications. Similarly, in patients with vagally related $\mathrm{AF}$ in whom increased parasympathetic tone has an important role in initiation and perpetuation of AF episodes, modulation of parasympathetic drive in addition to PVI seems to confer increased arrhythmia free survival ${ }^{(2,13)}$.

The exclusion of sinus and AV node intrinsic disease can be done non-invasively with Holter monitoring and exercise treadmill test. Maintained circadian variation of HR and normal chronotropic response to exercise are excellent surrogates of normal intrinsic sinus and AV node function. Previous studies have found comparable accuracy of Holter monitoring and treadmill exercise test with intrinsic HR measurements after pharmacological autonomic blockade. On the other hand, interpreting the measurement of baseline sinus node recovery time (SNRT) performed at electrophysiological study is difficult, as pacing suppression can be a normal phenomenon even in patients with normal sinus node function and because of existent overlap in recovery times between patients with normal and abnormal sinus node function. Additionally, it has sensitivity of only $70 \%{ }^{(14)}$. Furthermore, performing SNRT without pharmacological blockade is misleading and do no reflect the intrinsic properties of sinus and AV node and that is the reason why we didn't performed SNRT.

In our study we included patients with suspected parasympathetic driven bradyarrhythmias, that showed adequate chronotropic response on non-invasive evaluation which otherwise would have indication for pacemaker implant due to the severity of the clinical symptoms or the rhythm disorder. Our results show that modulation of parasympathetic cardiac system is effective in treating functional bradyarrhythmias. It has shown good results in immediate period after ablation with increase in HR, in shortening of AH intervals and WBCL and on follow up, as none of the patients had recurrence of symptoms or severe bradyarrhythmias.

So far, targeting GP for parasympathetic modulation has been quite challenging. Most of previous studies have used complicated methodologies requiring appropriate specific systems for identification of GP site, leading to extensive ablation in the atria and predisposing patients to iatrogenic atrial arrhythmias (15).

Previous works $(3,5,8,9,16,17)$ have shown that GP ablation can be achieved either using high frequency stimulation (HFS) (from epicardial or endocardial sides) to identify the GP or with an anatomical approach at known GP sites. Some works ${ }^{(3,9,12,18)}$ have posteriorly shown that the sensitivity of HFS is low $(21-71 \%)$ for the identification of GP sites and for assessment of the success of ablation. It is increasingly supported in the literature, the superiority of the anatomical approach over the HFS methodology due do the inconsistent parasympathetic response of the later. Besides, it allows avoidance of additional dedicated catheters and generators to perform HFS and spares the awake patient the unpleasant procedure ${ }^{(7,17,19)}$. Our work pursued a simplified methodology to perform parasympathetic modulation, using only an anatomical approach with 3D electroanatomic mapping and geometry reconstruction. With this method the GP described location was easily accessible, as was precise delivery of radiofrequency lesions with homogenization of the created scars and reducing potential additional procedure complications, without compromising success. Likewise, endpoints of the procedure are not standardized and there is no current consensus on endpoints for successful procedures. The most used assessment of parasympathetic modulation by achieving a blunted HR response with atropine administration after ablation has low value because of the increase in sympathetic tone ${ }^{(8,19)}$. So, in our study the endpoint of ablation was only anatomical deployment of lesion in pre specified sites and with pre specified settings, irrespective of any change in electrophysiological parameters.

Anatomic and physiological studies demonstrate that parasympathetic drive is predominantly located in the GP between the right superior pulmonary vein and right atria (which has most influence in cardiac parasympathetic innervation) and that the group of fibers located between the inferior vena cava and the right and left atria plays a major role in AV nodal innervation ${ }^{(4)}$. Increasing clinical evidence, have also shown that the most important modification of parasympathetic autonomic response during ablation occurs by targeting the right GP and some authors additionally claim predominant modulation from the right atria side of GP, namely during ablation of the right side of the interatrial septum along the coronary sinus 
$\operatorname{ostium}^{(1,5,20)}$. We used a simple and consistent method for GP ablation aiming at just the right GP with anatomical endpoints. Our results have shown that this strategy is feasible and effective, with less scar creation. In our patients, approaching the right GP alone seemed to be enough leading to abolishment of vagal cardiac tone, with significant shortening of RR and AH intervals and WBCL acutely. Also, we report a significant increase in minimal and mean heart rate 30 days after parasympathetic modulation, rendering patients asymptomatic with persistent effect in the medium-term. This data demonstrates that in the absence of standardized hard endpoints for GP ablation, an anatomic endpoint as surrogate of parasympathetic modulation and for simplification of the procedure purposes, with creation of a lesion subset in pre specified anatomic areas is enough.

\section{Conclusions:}

In this study, our results show that cardiac parasympathetic modulation is an effective alternative therapeutic in selected patients with severe functional bradyarrhythmias and that a simplified purely anatomic ablation strategy seems to be a valid approach.

\section{Study limitations:}

This is a study with a small number of patients and therefore larger prospective studies are needed to validate the strategy of performing ablation of only the right GP with a purely anatomic technique. Interestingly, it would be of value a larger study aiming at ablation of just the interatrial septum, for it seems to be the most effective site for cardiac parasympathetic denervation among these patients.

In spite no immediate effect on HR was noted during PVI in the 4 patients that had ablation for AF, we cannot exclude a possible additional effect of concurrent inadvertent left GP ablation in these patients.

Likewise, the long term effects of cardiac parasympathetic denervation are unknown and there are reports of coronary artery spasm or ventricular tachycardia after ablation ${ }^{(15)}$, probably by means of increased sympathetic tone, and it would be of utmost importance a larger study with longer follow up times in order to understand the safety outcomes of these procedures.

\section{Acknowledgements:}

The authors wish to thank Lia Marques (Abbot Inc.) for the help in assembling 3D electroanatomic maps.

\section{Data availability:}

The data underlying this article will be shared on reasonable request to the corresponding author.

\section{References:}

1. Caló L, Rebecchi M, Sciarra L, Luca L, Fagagnini A, Zuccaro L, et al. Catheter Ablation of Right Atrial Ganglionated Plexi in Patients with Vagal Paroxysmal Atrial Fibrillation. Circ Arrhythm Electrophysiol 2012; 5:22-31.

2. Choi E, Zhao Y, Everett T, Chen P. Ganglionated plexi as neuromodulation targets for atrial fibrillation. J Cardiovasc Electrophysiol 2017; 28: 1485-1491.

3. Scanavacca M, Pisani C, Hachul D, Lara S, Hardy C, Darrieux F, et al. Selective Atrial Vagal Denervation Guided by Evoked Vagal Reflex to Treat Patients With Paroxysmal Atrial Fibrillation. Circulation 2006; 114: 876-885.

4. Pachon J, Pachon E, Pachon J, Lobo T, Pachon M, Vargas R, et al. "Cardioneuroablation:" a new treatment for neurocardiogenic syncope, functional AV block and sinus dysfunction using catheter RF-ablation. Europace 2005; 7, 1-13.

5. Rivarola E, Hachul D, Wu T, Pisani C, Hardy C, Raimundi F, et al. Targets and End Points in Cardiac Autonomic Denervation Procedures. Circ Arrhythm Electrophysiol 2017; 10e004638: 1-9.

6. Mulpuru S, Shen W. Selective Modulation of the Cardiac Autonomic Nervous System, A New Strategy for Treatment of Cardioinhibitory Syncope. Circ Arrhythm Electrophysiol 2017; 10e004994: 1-3. 
7. Sun W, Zheng L, Qiao Y, Shi R, Hou B, Wu L, et al. Catheter Ablation as a Treatment for Vasovagal Syncope: Long-Term Outcome of Endocardial Autonomic Modification of the Left Atrium. Am Heart Assoc 2016; 5e003471: 1-10.

8. Pachon J, Pachon E, Santillana T, Lobo T, Pachon C, Pachon J. Simplified Method for Vagal Effect Evaluation in Cardiac Ablation and Electrophysiological Procedures. J Am Coll Cardiol EP 2015; 1: 451-60.

9. Lemery R, Birnie D, Tang A, Green M, Gollob M. Feasibility study of endocardial mapping of ganglionated plexuses during catheter ablation of atrial fibrillation. Heart Rhythm 2006; 3: $387-396$.

10. Brignole M, Auricchio A, Baron-Esquivias G, Bordachar P, Boriani G, Breithardt O, et al. 2013 ESC Guidelines on cardiac pacing and cardiac resynchronization therapy. Eur Heart J 2013; 34:2281-2329.

11. Katritsis G, Katritsis D. Cardiac Autonomic Denervation for Ablation of Atrial Fibrillation. Arrhythmia \& Electrophysiology Review 2014; 3:113-5.

12. Stavrakis S, Po S. Ganglionated Plexi Ablation: Physiology and Clinical Applications. Arrhythmia \& Electrophysiology Review. 2017; 6:186-90.

13. Pokushalov E, Romanov A, Artyomenko S, Shirokova N, Karashov A, Katritsis D, Karashov A, et al. Ganglionated Plexi Ablation Directed by High-Frequency Stimulation and Complex Fractionated Atrial Electrograms for Paroxysmal Atrial Fibrillation. PACE 2012; 35: 776-884.

14. Fogoros R. Electrophisiologic Testing. Blackwell Publishing, Pittsburgh PA; $4^{\text {th }}$ ed 2006: 61-69.

15. Qin M, Zeng C, Liu X. The cardiac autonomic nervous system: A target for modulation of atrial fibrillation. Clinical Cardiology 2019; 42:644-652.

16. Piotrowski R, Baran J, Kulakowski P. Cardioneuroablation using an anatomical approacj: a new and promising method foir the treatment of cardioinhibitory neurocardiogenic syncope. Kardiologia Polska 2018; 76: 1736-1738.

17. Katritis D, Giazitzoglou E, Sougiannis D, Goumas N, Paxinos G, Camm A. Anatomic Approach for Ganglionated Plexi Ablation in Patients With Paroxysmal Atrial Fibrillation. Am J Cardiol 2008; 102: 330-334.

18. Pokushalov E, Romanov A, Shugayev P, Artyomenko S, Shirokova N, Turov D, et al. Selective ganglionated plexi ablation for paroxysmal atrial fibrillation. Heart Rhythm 2009; 6: 1257-1264.

19. Osório T, Paparella G, Stec S, Chierchia G, Asmundis C. Cardiac parasympathetic modulation in the setting of radiofrequency ablation for atrial fibrillation. Arch Med Sci. 2019; 84717.

20. Hu F, Zheng L, Liang E, Ding L, Wu L, Chen G, et al. Right anterior ganglionated plexus: The primary target of cardioneuroablation? Heart Rhythm 2019; 16: 1545-1551.

\section{Tables}

Table 1. Baseline Clinical Characteristics of the cohort.

\begin{tabular}{|c|c|c|c|c|c|c|}
\hline Patient & Gender & Age (years) & Diagnosis & Symptoms & AFib & Other \\
\hline 1 & Male & 51 & $\begin{array}{l}\text { SB and } \\
\text { Sinus Arrest }\end{array}$ & Syncope & No & Athlete \\
\hline 2 & Female & 34 & $\begin{array}{l}\text { High grade } \\
\text { AVB }\end{array}$ & None & No & $\longrightarrow$ \\
\hline 3 & Male & 55 & $\begin{array}{l}\text { SB and } \\
\text { Sinus Arrest }\end{array}$ & Syncope & Yes & Athlete \\
\hline 4 & Male & 58 & $\begin{array}{l}\text { SB and } \\
\text { Sinus Arrest }\end{array}$ & Pre syncope & Yes & - \\
\hline 5 & Female & 69 & $\begin{array}{l}\text { Cardioinhibitory } \\
\text { syncope }\end{array}$ & Syncope & Yes & - \\
\hline 6 & Female & 63 & Sinus Arrest & None & No & OSA \\
\hline 7 & Male & 70 & $\begin{array}{l}\text { SB and } \\
\text { Sinus Arrest }\end{array}$ & Pre syncope & Yes & \\
\hline
\end{tabular}




\begin{tabular}{|c|c|c|c|c|c|c|}
\hline Patient & Gender & Age (years) & Diagnosis & Symptoms & AFib & Other \\
\hline 8 & Male & 48 & $\mathrm{SB}$ & Pre syncope & No & $\begin{array}{l}\text { Athlete; PM } \\
\text { explantation } \\
\text { (device } \\
\text { infection) }\end{array}$ \\
\hline 9 & Male & 20 & Sinus Arrest & Syncope & No & Athlete \\
\hline 10 & Male & 42 & $\begin{array}{l}\text { Sinus Arrest } \\
\text { and high } \\
\text { grade AVB }\end{array}$ & None & No & Athlete \\
\hline 11 & Male & 47 & Sinus Arrest & None & No & $\longrightarrow$ \\
\hline 12 & Male & 19 & $\begin{array}{l}\text { Cardioinhibitory } \\
\text { syncope }\end{array}$ & Syncope & No & $\longrightarrow$ \\
\hline 13 & Male & 68 & $\begin{array}{l}\text { High grade } \\
\text { AVB }\end{array}$ & Pre Syncope & No & Athlete \\
\hline
\end{tabular}

(SB - Sinus Bradycardia; AVB - Atrioventricular Block; OSA - Obstructive Sleep Apnea; PM - Pacemaker)

Table 2. Electrocardiographic characteristics of the cohort, before and after ablation.

\begin{tabular}{|c|c|c|c|c|}
\hline Patient & Longest Pause (ms) & Basal ECG HR (bpm) & 24h-Holter minimal HR, pre Abl (bpm) & 24h-Holter minimal HR \\
\hline 1 & 8320 & 45 & 21 & 70 \\
\hline 2 & 7000 & 58 & 45 & 60 \\
\hline 3 & 4000 & 40 & 26 & 50 \\
\hline 4 & 5350 & 76 & 36 & 51 \\
\hline 5 & 10000 & 55 & 43 & 48 \\
\hline 6 & 13200 & 88 & 39 & 43 \\
\hline 7 & 3300 & 35 & 46 & 41 \\
\hline 8 & 4320 & 42 & 26 & 31 \\
\hline 9 & 3300 & 61 & 14 & 62 \\
\hline 10 & 3650 & 45 & 12 & 46 \\
\hline 11 & 9000 & 75 & 42 & 42 \\
\hline 12 & 18000 & 60 & 52 & 56 \\
\hline 13 & 10000 & 49 & 42 & 43 \\
\hline
\end{tabular}

(ms - milliseconds; ECG - Electrocardiogram; HR - Heart Rate; bpm - beats per minute; Abl - Ablation)

Table 3. Electrophysiological characteristics of the cohort, before and after ablation and follow-up.

\begin{tabular}{|c|c|c|c|c|c|c|c|c|c|c|c|}
\hline Patient & $\begin{array}{l}\text { RR pre } \\
\mathrm{Abl} \\
(\mathrm{ms})\end{array}$ & $\begin{array}{l}\mathrm{RR} \\
\text { post } \\
\text { Abl } \\
(\mathrm{ms})\end{array}$ & $\begin{array}{l}\text { RR } \\
\text { short- } \\
\text { ening } \\
(\%)\end{array}$ & $\begin{array}{l}\text { HR } \\
\text { Accl } \\
(\mathrm{bpm})\end{array}$ & $\begin{array}{l}\text { AH pre } \\
\mathrm{Abl} \\
(\mathrm{ms})\end{array}$ & $\begin{array}{l}\text { AH } \\
\text { post } \\
\text { Abl } \\
(\mathrm{ms})\end{array}$ & $\begin{array}{l}\text { AH } \\
\text { short- } \\
\text { ening } \\
(\%)\end{array}$ & $\begin{array}{l}\text { WBCL } \\
\text { pre Abl } \\
(\mathrm{ms})\end{array}$ & $\begin{array}{l}\text { WBCL } \\
\text { post } \\
\text { Abl } \\
(\mathrm{ms})\end{array}$ & $\begin{array}{l}\text { WBCL } \\
\text { short- } \\
\text { ening } \\
(\%)\end{array}$ & PVI \\
\hline 1 & 1395 & 1000 & 28.3 & 17 & 130 & 50 & 61,5 & 450 & 400 & 11.1 & No \\
\hline 2 & 1035 & 600 & 42.0 & 42 & 115 & 115 & 0 & 540 & 440 & 18.5 & No \\
\hline 3 & 1200 & 800 & 33.3 & 25 & 110 & 70 & 36,4 & 460 & 430 & 6.5 & Yes \\
\hline 4 & 732 & 652 & 10.9 & 10 & 115 & 115 & 0 & 450 & 440 & 2.2 & Yes \\
\hline 5 & 1539 & 606 & 60.6 & 60 & 125 & 110 & 12 & 480 & 460 & 4.2 & Yes \\
\hline 6 & 1395 & 1017 & 27.1 & 16 & 105 & 85 & 19 & 420 & 400 & 4.8 & No \\
\hline 7 & 1395 & 833 & 40.3 & 29 & 95 & 80 & 15,8 & 510 & 480 & 5.9 & Yes \\
\hline
\end{tabular}




\begin{tabular}{|c|c|c|c|c|c|c|c|c|c|c|c|}
\hline Patient & $\begin{array}{l}\text { RR pre } \\
\text { Abl } \\
(\mathrm{ms})\end{array}$ & $\begin{array}{l}\mathrm{RR} \\
\text { post } \\
\text { Abl } \\
(\mathrm{ms})\end{array}$ & $\begin{array}{l}\text { RR } \\
\text { short- } \\
\text { ening } \\
(\%)\end{array}$ & $\begin{array}{l}\text { HR } \\
\text { Accl } \\
(\mathrm{bpm})\end{array}$ & $\begin{array}{l}\text { AH pre } \\
\text { Abl } \\
(\mathrm{ms})\end{array}$ & $\begin{array}{l}\mathrm{AH} \\
\text { post } \\
\text { Abl } \\
(\mathrm{ms})\end{array}$ & $\begin{array}{l}\text { AH } \\
\text { short- } \\
\text { ening } \\
(\%)\end{array}$ & $\begin{array}{l}\text { WBCL } \\
\text { pre Abl } \\
(\mathrm{ms})\end{array}$ & $\begin{array}{l}\text { WBCL } \\
\text { post } \\
\text { Abl } \\
(\mathrm{ms})\end{array}$ & $\begin{array}{l}\text { WBCL } \\
\text { short- } \\
\text { ening } \\
(\%)\end{array}$ & PVI \\
\hline 8 & 984 & 723 & 26.5 & 22 & 95 & 85 & 10,5 & 800 & 600 & 25.0 & No \\
\hline 9 & 769 & 690 & 10.3 & 9 & 95 & 71 & 25,3 & 450 & 370 & 17.8 & No \\
\hline 10 & 1818 & 923 & 49.2 & 32 & 140 & 90 & 35,7 & 1000 & 620 & 38.0 & No \\
\hline 11 & 934 & 698 & 25.6 & 22 & 122 & 50 & 59,0 & 400 & 340 & 15.0 & No \\
\hline 12 & 882 & 600 & 32.0 & 32 & 115 & 93 & 19,1 & 440 & 410 & 6.8 & No \\
\hline 13 & 1111 & 882 & 20.6 & 14 & 115 & 105 & 8,7 & 440 & 370 & 15.9 & No \\
\hline
\end{tabular}

(RR - RR intervals; ms - milliseconds; HR Accl - Heart Rate acceleration; AH - A to His interval; WBCL - Wenckebach cycle length; Abl - Ablation; PVI - Pulmonary Vein Isolation; FUP - Follow Up)

\section{Figures}

Figure 1. Electroanatomic 3D endocardial mapping cluster ablation lesions set at right anterior and inferior GP, approach from the left atrium

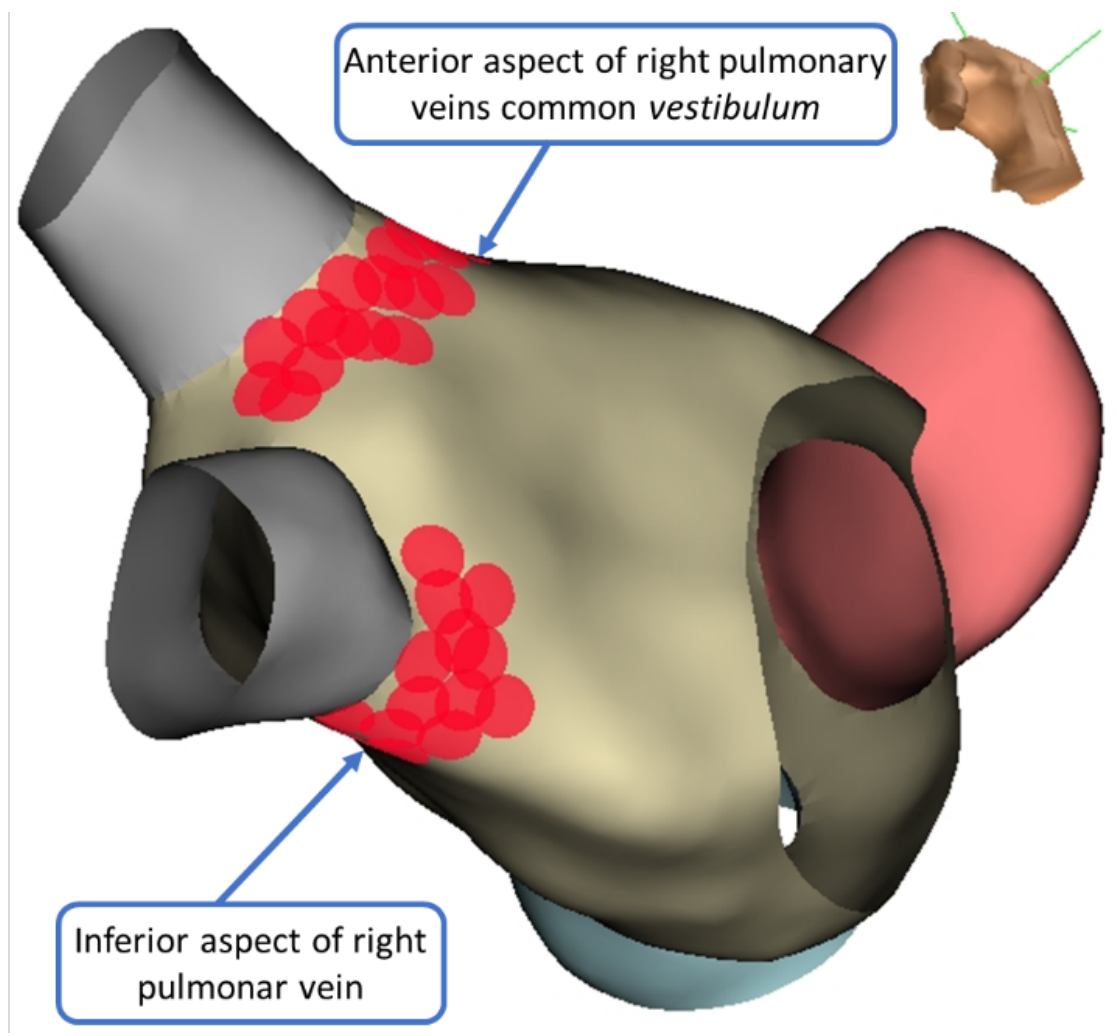

Figure 2. Electroanatomic 3D endocardial mapping showing cluster ablation lesions set from a right atrium approach, at the superior vena cava junction and posterior aspect of interatrial septum 


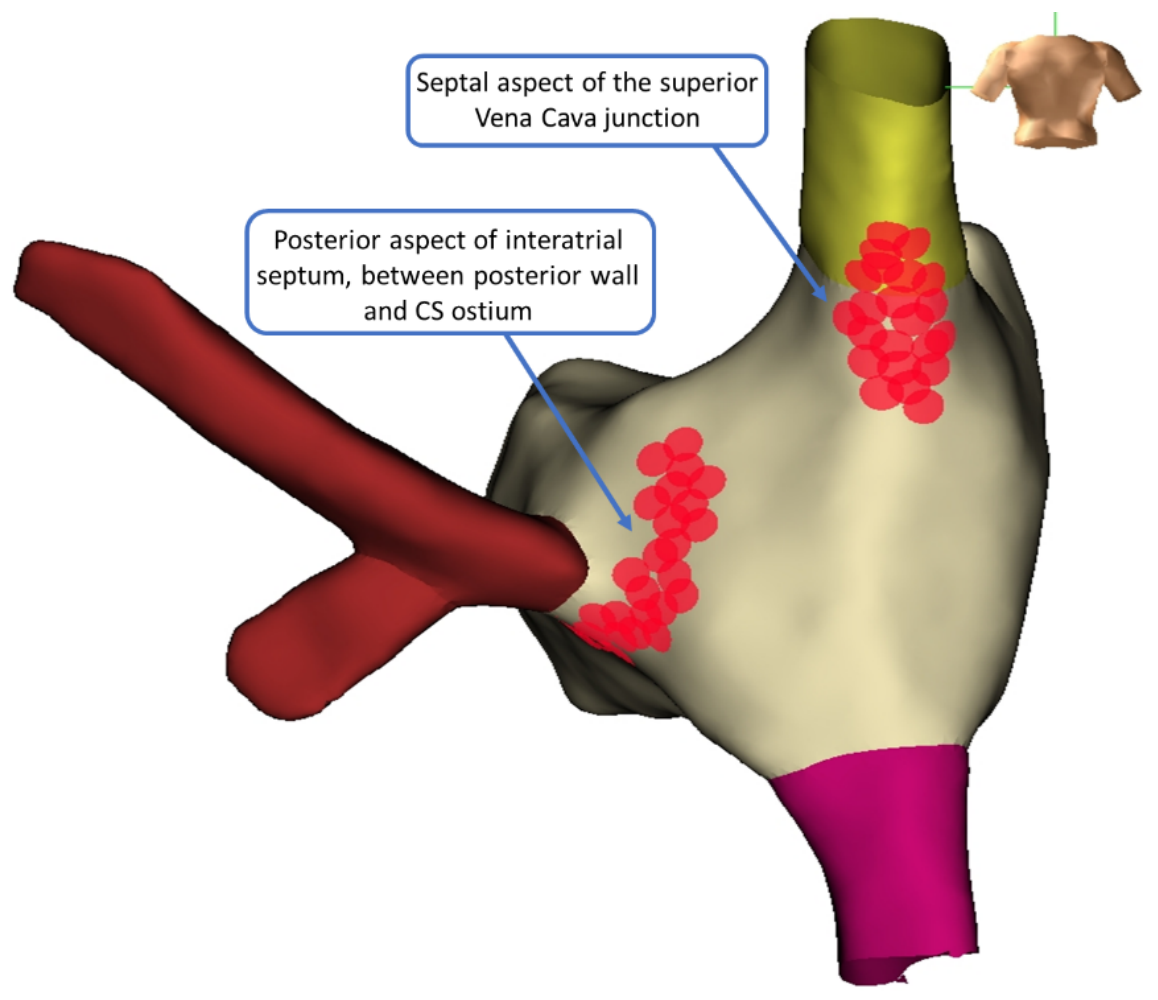

Figure 3 (representative figure). Electroanatomic 3D endocardial mapping showing cluster ablation lesions in the opposite areas of LA and RA, targeting anterior right and inferior right GP

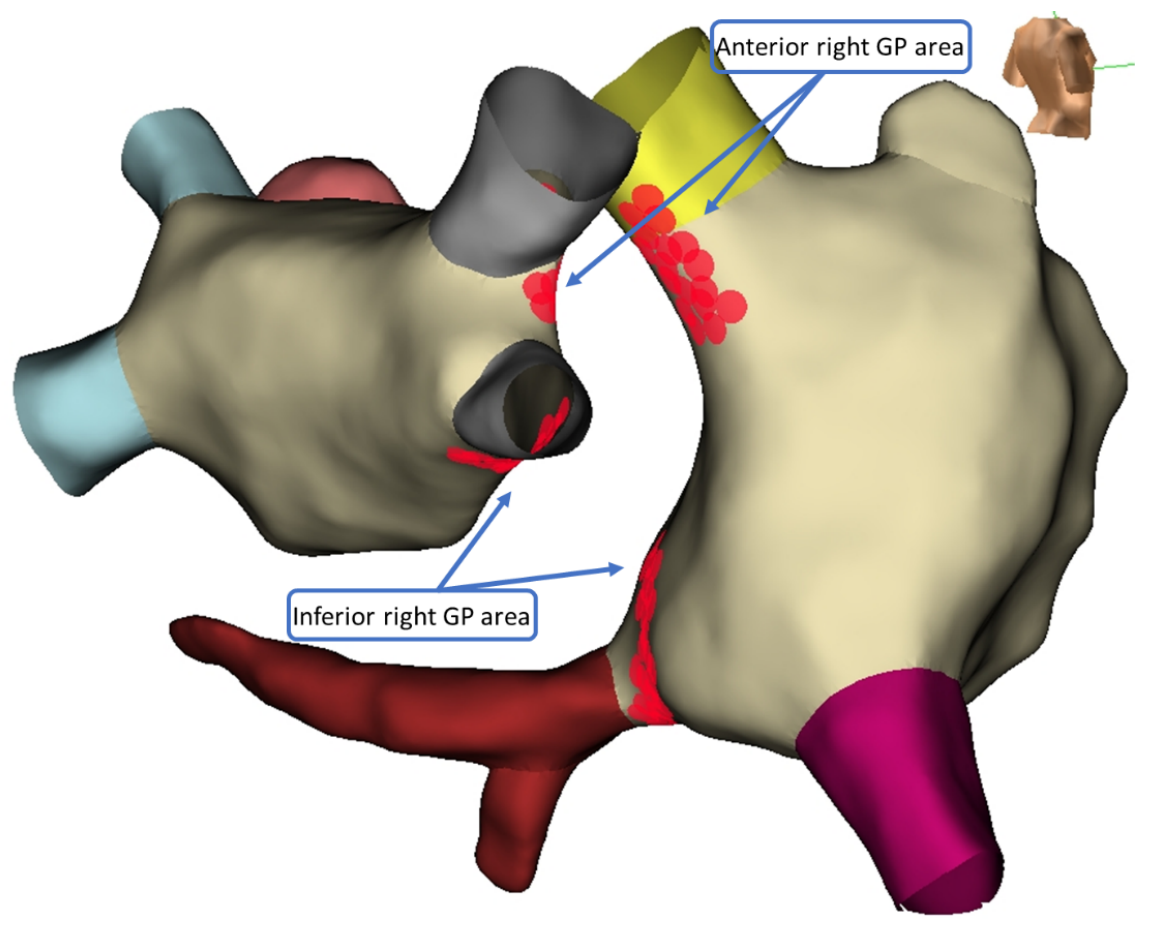


Figure 4. Effect on RR intervals after ablation of GP from a right atrium approach at the posterior aspect of the interatrial septum, patient \#5; superior image - basal RR intervals; inferior image - RR intervals after ablation

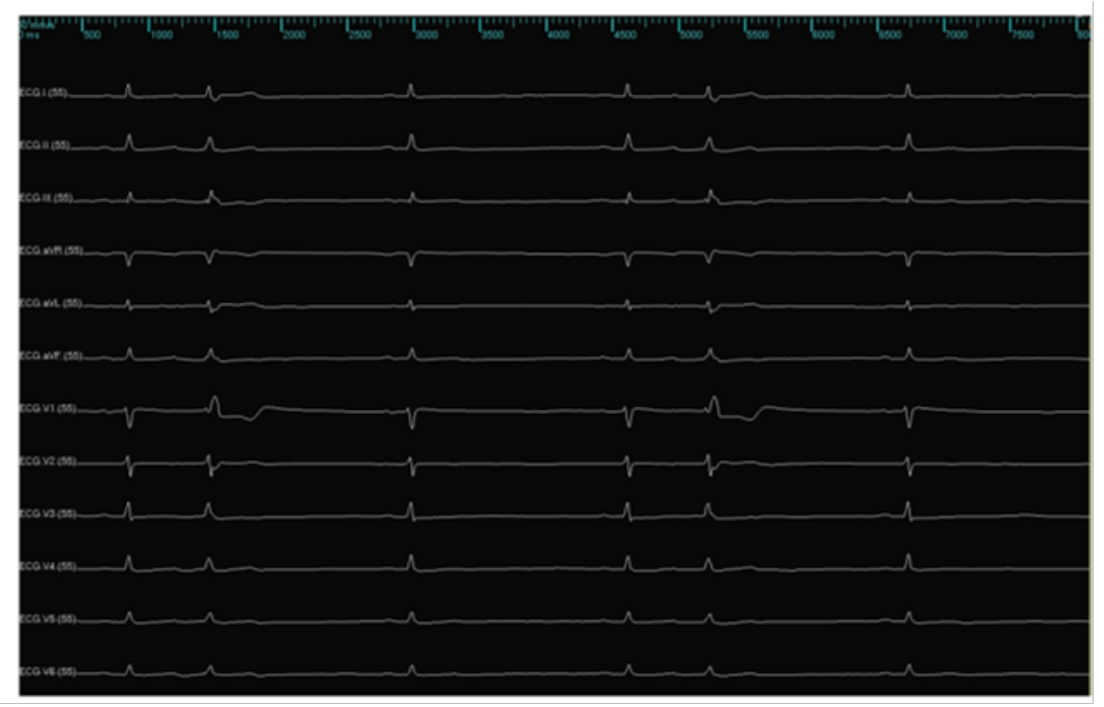

\section{ECG after RA GP modulation}

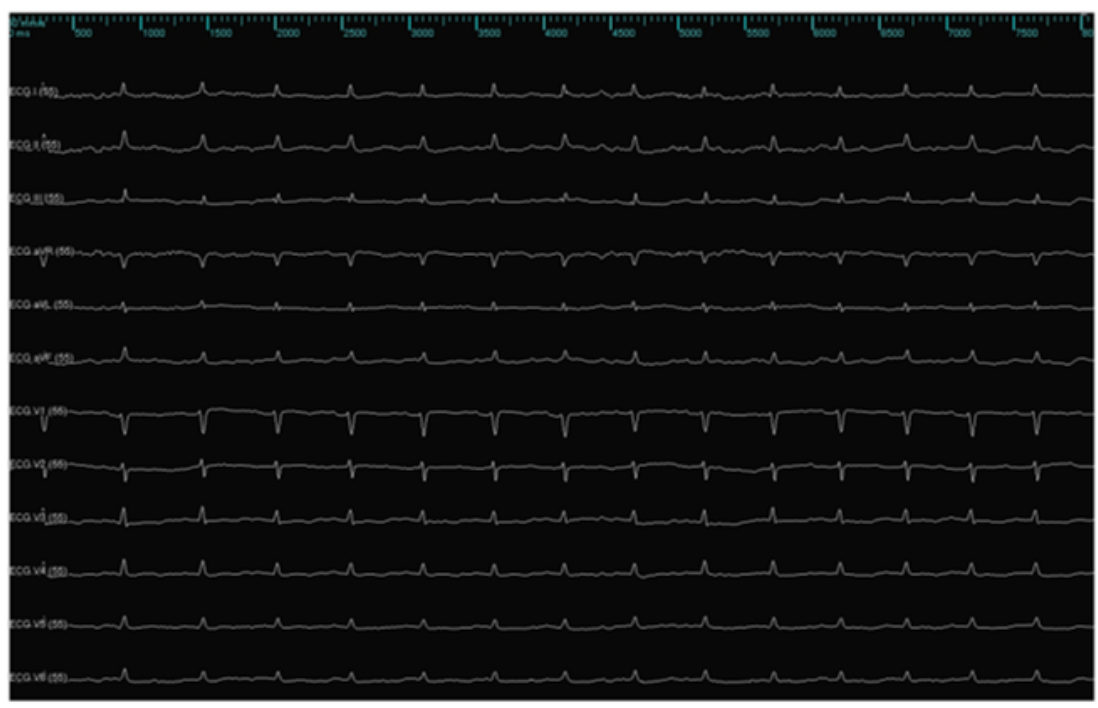

